\title{
When a Chance to Cut Is Not a Chance to Cure: A Future for Palliative Surgery?
}

\author{
Paul J. Mosca, MD, PhD', Dan G. Blazer III, MD', Jane L. Wheeler, MSPH ${ }^{2}$, and Amy P. Abernethy, MD $^{2}$ \\ ${ }^{1}$ Division of Surgical Oncology, Department of Surgery, Duke University Medical Center, Durham, NC 27710; ${ }^{2}$ Division \\ of Medical Oncology, Duke University Medical Center, Durham, NC 27710
}

\begin{abstract}
In the context of healthcare reform, Surgery stands at a critical juncture. Attempting to rein in healthcare spending, legislators and payers can be expected to closely examine the legitimacy and necessity of a variety of medical treatments, including surgical procedures. Among these procedures, the most at risk for dismissal based on perceived ineffectiveness or lack of need may be those performed near the end of life, when the potential benefit of surgical intervention may seem negligible. While procedures may be performed for a variety of reasons toward the end of life-some indeed being inappropriate and/or unnecessary-palliative surgery plays an important role in the management of incurable disease. The purposes of this article are to: describe the place for palliative surgery in the armamentarium of palliative care; discuss potential challenges to patients' access to palliative surgery that may arise from health policy or quality initiatives based on poor evidence; and outline a strategy for (a) systematically differentiating palliative surgeries from other, potentially expendable surgeries performed near the end of life, and (b) defining a plan for generating the evidence base to support best practice.
\end{abstract}

Palliative care, encompassing palliative surgery, seeks to reduce the severity of disease-related symptoms, rather than to halt or reverse progression of the disease itself. Its goals are to prevent and relieve suffering and to improve quality of life for people facing advanced illness. Surgical

\footnotetext{
(C) Society of Surgical Oncology 2011

Published Online: 17 May 2011

P. J. Mosca, MD, PhD

e-mail: paul.mosca@duke.edu
}

procedures occurring toward the end of life may be performed for a variety of reasons, but many are palliative in nature in that they are not expected to be curative. Some studies have shown that, while associated with significant morbidity, palliative surgery can positively impact quality of life in properly selected patients with advanced cancer. ${ }^{1-3}$ However, as yet, there remains a lack of standards, clear definitions, or consensus regarding optimal use of palliative surgery for symptom control.

Though the intent of palliative surgery is to alleviate suffering, its role (Table 1) in end-of-life care is often very difficult to assess for patients and practitioners alike. First, there is the practical issue of defining "end of life" as a period of care. During this time, there is substantial risk of perioperative morbidity or mortality, and postoperative pain and other symptomatology (particularly when there are procedure-related complications) can actually worsen, rather than improve, quality of life. For late-stage patients, palliative surgical procedures may be very costly, contributing to the extraordinary monetary resources spent in the last few months of life. However, in this amorphous period termed "end of life," there are also clear palliative benefits of appropriately selected and safely conducted procedures.

Unfortunately, despite anecdotal evidence that, when used appropriately, palliative surgeries can ameliorate symptoms and enhance quality of life, there is little highquality published evidence regarding the appropriate role, type, and timing of surgical procedures near the end of life. Clarification, through research, of the role and parameters of palliative surgery has met various challenges. Tensions surrounding the ethics and costs of research performed near the end of life have impeded the initiation of studies, and have created a barrier to recruitment of eligible patients. Due to the poor quality of most research on palliative surgery, typical of palliative care more generally, we lack a robust evidence base that can guide clinicians regarding 
TABLE 1 Examples of palliative procedures performed near the end of life

\begin{tabular}{|c|c|}
\hline Nature of procedure & Examples \\
\hline Symptom relief for incurable benign disease & $\begin{array}{l}\text { Excision of large condylomata } \\
\text { Procedures (e.g., banding of esophageal varices or shunt procedures) for end-stage liver } \\
\text { disease }\end{array}$ \\
\hline $\begin{array}{l}\text { Symptom relief for incurable malignant disease: } \\
\text { nonresectional }\end{array}$ & $\begin{array}{l}\text { Biliary and/or gastric bypass for unresectable, obstructing periampullary cancers } \\
\text { Chemical splanchnicectomy for intractable pain (e.g., locoregionally advanced pancreatic } \\
\text { cancer) } \\
\text { Intestinal bypass or ostomy for malignant bowel obstruction } \\
\text { Decompressing gastrostomy tube placement for malignant bowel obstruction } \\
\text { Peritoneal dialysis catheter for drainage of recurrent malignant ascites }\end{array}$ \\
\hline $\begin{array}{l}\text { Symptom relief for incurable malignant disease: } \\
\text { resectional/debulking (cytoreductive) procedure }\end{array}$ & $\begin{array}{l}\text { Resection of primary tumor or metastatic lesion(s) for pain, obstruction, and/or bleeding in } \\
\text { setting of unresectable distant disease (e.g., palliative mastectomy, resection of primary } \\
\text { tumor in stage IV colorectal cancer) } \\
\text { Resection/debulking of symptomatic carcinoid tumor }\end{array}$ \\
\hline $\begin{array}{l}\text { Intended prolongation of survival: resectional/ } \\
\text { debulking procedure }\end{array}$ & $\begin{array}{l}\text { Debulking of advanced ovarian cancer } \\
\text { Metastasectomy or ablation of advanced cancer with incomplete or suboptimal treatment } \\
\text { (e.g., R2 - gross disease remaining) }\end{array}$ \\
\hline
\end{tabular}

indications for and application of surgical procedures in patients with advanced, life-limiting disease. While this evidence gap will make it difficult to differentiate between appropriate versus inappropriate palliative surgical procedures near the end of life, it does not obviate the need for that delineation.

Attempts to distinguish the purposes of palliative surgery from those of curative surgery have actually confused rather than clarified the role of palliative surgery. In a recent New England Journal of Medicine article, Temel and colleagues demonstrated that early palliative care provided in conjunction with usual oncology care for people with non-small cell lung cancer improved quality of life and mood, reduced healthcare expenses, and significantly prolonged survival. ${ }^{4}$ Similarly, while the primary goal of surgery in the setting of incurable disease remains symptom management, surgery in this setting may also prolong disease-free or overall survival, and this survival benefit may (or may not) be associated with improved quality of life. Resectional or debulking surgery, for example, may be indicated for certain patients with incurable, metastatic malignancy. In the case of metastatic breast cancer, surgical extirpation of the primary tumor may improve survival, though this conclusion remains controversial. ${ }^{5}$ Similarly, a small retrospective study recently found that resection of the primary site of metastatic colorectal cancer may be associated with improved survival compared with palliative bypass, colostomy, or nonoperative management alone. ${ }^{6}$ Prospective data from high-quality clinical trials are needed to establish the impact of surgery performed near the end of life on outcomes that are traditionally associated with both palliative and curative approaches.

\section{PALLIATIVE SURGERY IN RELATION TO QUALITY METRICS}

The increasing focus on quality of care continues under healthcare reform. Energized by the Institute of Medicine's seminal 1999 publication, To Err is Human: Building a Safer Health System, increasing attention has been placed on safety and quality in healthcare. ${ }^{7}$ This initiative could greatly benefit patients and other stakeholders. Certainly, medical errors represent a significant quality issue in Surgery as well as in other specialties; yet even "low-hanging fruit"-such as eliminating wrong-site surgeries-has proven difficult, despite strict policies and procedures. Other efforts aimed at tracking and incentivizing the application of evidence-based treatment protocols have also presented significant challenges. A more fundamental issue-which surgical procedures should be performed in which clinical situations - is likely to receive increasing attention because of the risks and costs associated with surgery, and public demand for more personalized care and value-based decision-making.

Optimizing the utilization of surgeries near the end of life presents a particularly complex and multidimensional problem. Some procedures - such as those that offer little or no palliative benefit, do not extend survival, and are associated with significant morbidity or financial costsindeed may be inappropriate for patients who are near the end of life. In these cases, the debate surrounding chemotherapy in the last 2 weeks of life provides an exemplar for consideration of surgical interventions. Rigorous analysis of available evidence suggested that chemotherapy delivered near the end of life is not related to likelihood of benefit; ${ }^{8}$ Based on these data-driven analyses, quality 
measures for surveillance of end-of-life care have been developed, and chemotherapy given within 14 days of death is among them. Specifically, the proportion of patients receiving chemotherapy in the last 2 weeks of life is a metric monitored in a number of quality improvement programs, including the American Society of Clinical Oncology's Quality Oncology Practice Initiative (QOPI); the targeted performance on this measure is to approach $0 \%$, though current performance is around $10 \%$.

Similar potential targets in palliative surgery may be challenging to define. These procedures have attendant risks and are not guaranteed to reduce symptoms, but they may significantly relieve suffering in the setting of advanced malignancy, and they have the potential to prolong survival. Their appropriateness and timing are not well defined. Studies to inform the evidence base and quality metrics for these procedures are critical to ensure their appropriate application for palliative purposes. The surgical community must advocate inclusion of certain patient-centered outcomes, such as distress or pain, in these studies alongside more obvious outcomes such as survival. Metrics developed to evaluate performance related to these procedures must take into account the full range of their benefits, including symptom reduction, without penalizing clinicians or institutions for expected outcomes in advanced disease, such as hospitalization or death.

The mantra in current discussions of quality of care is "getting the right care to the right patient at the right time." With palliative surgery, the definition of the "right care" needs to be closely aligned with intent. One cannot assume that all procedures performed near the end of life are inappropriate; this assumption would lead to underutilization of surgical procedures that help achieve the goals of palliative care (reducing suffering and optimizing quality of life). Conversely, one cannot assume that every surgery conducted at the end of life has palliative intent. Lacking a taxonomy of palliative surgical procedures that defines their appropriate uses in the context of intent, we run the risk of palliative surgeries being evaluated against metrics that are not aligned with their intent, in particular metrics based on financial rather than patient-centered objectives, resulting in determination that palliative procedures are inappropriate and/or ineffective.

Historically, metrics designed to evaluate-and hopefully improve-quality in surgery have primarily focused on measures of morbidity and mortality. Initiatives such as the National Surgical Quality Improvement Program (NSQiP) promote utilization of practical metrics and benchmarks for improving surgical quality. While early studies have not consistently demonstrated a positive impact on outcomes, it appears that through participation in these initiatives hospitals may improve their performance as measured by some quality metrics. 9 Incentives, however, must be carefully crafted. For example, beginning in October 2012, federal regulators will withhold $1 \%$ of all inpatient Medicare payments (with a rising percentage each subsequent year) to hospitals at which 30-day readmission rates for patients with certain chronic conditions fall above the national average. Paradoxically, this incentive may result in longer initial lengths of stay and corresponding unnecessary costs.

Among a host of tools being developed for tracking and reporting on healthcare quality indicators, the physician report card is gaining momentum in Surgery. Interest in this approach reflects justified concern about the safety, quality, and cost of surgical procedures-particularly if inappropriately utilized-and the increasing scrutiny of a large number of stakeholders interested in the use of quality metrics to facilitate quality improvement. ${ }^{10,11}$ In practice, active monitoring of surgical outcomes itself may positively impact quality metrics, possibly due to the Hawthorne effect. ${ }^{12}$ Additional incentives based on quality metrics, such as pay-for-performance policies, may also influence a surgeon's practices. Making a particular surgeon's data known to him/her may encourage a surgeon to focus on improving those areas in which he/she lags behind benchmarks. However, using report cards to compare physicians with one another could have a deleterious impact on appropriate provision of palliative surgery. For example, a palliative operation performed to reduce suffering in a patient nearing the end of life, followed by the patient's expected death, could be recorded as an unanticipated adverse event that is equivalent to a mortality occurring after a therapeutic oncologic surgical procedure. Neglecting to account for the intent of care, this sort of quality metric would serve as a strong disincentive to perform palliative surgery. Simply put, basic quality metrics such as mortality can be misleading and may set up incentives that are harmful to patients with advanced disease, unless they are designed to factor in the intent of the intervention.

\section{PALLIATIVE SURGERY AND HEALTH POLICY}

Now is the time for Surgery to clarify the role and appropriate use of its procedures in order to ensure that new healthcare policies based on emerging quality metrics preserve patients' access to beneficial surgical interventions. Nowhere is this more important than in defining appropriate versus inappropriate use of palliative surgeries for patients near the end of life. For over two decades, consistently $25 \%$ or more of Medicare funds have been expended on patients within the last year of life. ${ }^{13}$ As concern about ballooning healthcare costs has escalated, consumer advocacy groups have (legitimately) become 
more worried about overmedicalization of the healthcare system. ${ }^{14}$ Consequently, overutilization metrics are increasingly prevalent. To extend the example of chemotherapy in Medical Oncology, use of chemotherapy within the last month of life is being used as a measure of quality in the context of QOPI.

While timing of surgical procedures with respect to the end of life-other than procedure-related mortality-is not yet tracked as a routine means of evaluating quality, the performance of possibly inappropriate procedures near the end of life will undoubtedly be subject to increasing scrutiny. An example is extensive cytoreductive surgery in the setting of advanced, incurable malignancy at a point when there is little hope of meaningful survival. However, we must not lose sight of the fact that certain surgeries performed in the last 6 months of life, such as pleurodesis for symptomatic malignant pleural effusions, may be important elements of the physician's armamentarium for symptom management. We must achieve a balance that settles the current tension between (a) ensuring that patients are not unnecessarily exposed to treatments that are not going to benefit them, and (b) ensuring that patients have access to interventions that could meaningfully reduce their suffering. Among the various stakeholders, surgeons are best prepared to advise on this balance.

\section{OPTIMIZING THE ROLE OF PALLIATIVE SURGERY IN HEALTHCARE}

Many surgeries performed at the end of life may not only be appropriate, but may contribute positively to the patient's quality of life. In order to establish a viable, systematic strategy for driving down use of inappropriate surgical procedures near the end of life, and for optimizing appropriate application of palliative procedures in particular, we first must develop a means of describing and differentiating among procedures performed in this setting. This taxonomy will entail both a structured framework for classifying surgical procedures performed near the end of life and a systematic method for quantifying these procedures and monitoring trends in utilization over time.

We are currently examining the type of and trend in frequency of surgical procedures performed at Duke University Hospital. Our preliminary taxonomy differentiates among procedures that: (1) directly cause death because of postoperative complications; (2) are performed to prevent death, but are not successful in doing so; (3) are part of the treatment plan, or are performed to address a condition incidentally occurring near the end of life (e.g., acute cholecystitis); (4) are performed with palliative intent; or (5) are performed without known or clearly documented intent.
As a first step, this study will characterize the procedures which we are performing (at one institution) near the end of life, the surrounding circumstances, and the factors that impact decision-making on the part of the healthcare team, patient, and family. This understanding will set the backdrop for future comparative effectiveness questions, such as whether a particular surgical intervention, medical intervention, or comfort measures alone would be the most clinically effective and cost-effective approach to symptom management in the palliative setting. If intent is understood, and if metrics accommodate that intent, then such questions will be suitable research topics answerable through the use of registries and other databases. Involvement of surgeons in this research will be key to the accurate description of palliative surgical procedures, their proper classification, and analysis of their comparative effectiveness in real-world contexts for defined purposes. The desired outcome of these studies would be optimal use of surgical interventions for palliative purposes where they are effective, and abstention from use of these procedures where they are not likely to benefit the patient.

\section{SIGNIFICANCE AND CHALLENGE}

Palliative surgery comprises some of the most invasive, risky, and costly procedures performed during the last few months of life, and hence they will likely be a major focus of comparative effectiveness research, as well as of fiscal scrutiny, in the imminent future. Quality metrics are almost certain to be developed to oversee the utilization of palliative surgery; the exact nature of these metrics will exert a strong force on medical decision-making, and thus on patients' access to palliative surgical interventions. Inherent in determining the purpose of these future metrics are significant challenges and complexities. One level of complexity resides in the numerous stakeholders involved in palliative surgery-from providers and patients to institutions, payers, and government-and in the related evaluative question, "better for whom?" Another level of complexity is definitional: What palliative surgical procedures are performed near the end of life? What is their respective intent? What factors lead to the decision to perform a given surgical procedure? What are the risk- and cost-benefit ratios of such interventions relative to other options?

The overarching challenge, and a mandate for healthcare, is that of ensuring that patients in need of appropriate palliative surgical procedures continue to receive them (Table 2). To this end, representatives of Surgery must actively participate in discussions regarding the development of metrics to determine the appropriate use of surgical procedures. Metrics based on financial impact or procedural 
TABLE 2 Proposed agenda for defining the role of palliative surgery

Retrospectively determine the types of and trends in frequency of palliative surgical procedures performed near the end of life

Retrospectively determine the intent of palliative surgical procedures and contrast this with the intent of other procedures performed near the end of life

Estimate costs associated with surgical procedures performed near the end of life, by intent and appropriateness

Develop prospective classification system denoting intent of procedures performed in patients with predicted survival of less than 6 months

Prospectively collect data regarding surgeries performed near the end of life, including palliative surgeries

Perform comparative effectiveness research about current palliative surgical interventions to develop evidence on which to base guidelines regarding the role, appropriateness, and cost-effectiveness of palliative surgical options

Ensure that quality metrics, health policies, and financial incentives reflect the intent of surgeries performed near the end of life

Create a prospective research agenda to develop and test new, better honed, palliative interventions and define their role in relation to currently available options

mortality as measures of quality — without adjusting for the performance of palliative procedures-could incentivize surgeons to avoid performing procedures that might provide significant symptom relief or quality-of-life improvement for patients near the end of life. We applaud the substantial efforts currently being devoted to differentiating between appropriate and inappropriate procedures, and the desire to discontinue paying for inappropriately utilized services. It is critical, however, to define what is "appropriate" with great care when services are being provided to patients near the end of life, lest surgeons be impeded from providing vital services and patients be deprived of care directed toward alleviation of their suffering.

\section{REFERENCES}

1. Miner TJ, Jaques DP, Shriver CD. A prospective evaluation of patients undergoing surgery for the palliation of an advanced malignancy. Ann Surg Oncol. 2002;9(7):696-703.

2. Podnos YD, Juarez G, Pameijer C, Choi K, Ferrell BR, Wagman LD. Impact of surgical palliation on quality of life in patients with advanced malignancy: results of the decisions and outcomes in palliative surgery (DOPS) trial. Ann Surg Oncol. 2007;14(2): 922-8.

3. Krouse RS, Nelson RA, Farrell BR, et al. Surgical palliation at a cancer center: incidence and outcomes. Arch Surg. 2001;136(7): 773-8.

4. Temel JS, Greer JA, Muzikansky A, et al. Early palliative care for patients with metastatic non-small-cell lung cancer. $N$ Engl $J$ Med. 2010;363(8):733-42.
5. Rapiti E, Verkooijen HM, Vlastos G, et al. Complete excision of primary breast tumor improves survival of patients with metastatic breast cancer at diagnosis. J Clin Oncol. 2006;24(18):2743-9.

6. Tanoue Y, Tanaka N, Nomura Y. Primary site resection is superior for incurable metastatic colorectal cancer. World J Gastroenterol. 2010;16(28):3561-6.

7. Kohn L, Corrigan J, Donaldson M, eds of the Committee on Quality of Health Care in America. To err is human: building a safer health system. Washington, DC: National Academy Press; 1999.

8. Earle CC, Landrum MB, Souza JM, Neville BA, Weeks JC, Ayanian JZ. Aggressiveness of cancer care near the end of life: is it a quality-of-care issue? J Clin Oncol. 2008;26(23):3860-6.

9. Khuri SF, Henderson WG, Daley J, et al. Successful implementation of the Department of Veterans Affairs' National Surgical Quality Improvement Program in the private sector: the Patient Safety in Surgery study. Ann Surg. 2008;248(2):329-36.

10. Glance LG, Dick AW, Mukamel DB, Li Y, Osler TM. How well do hospital mortality rates reported in the New York State CABG report card predict subsequent hospital performance? Med Care. 2010;48(5):466-71.

11. Epstein AJ. Effects of report cards on referral patterns to cardiac surgeons. J Health Econ. 2010;29(5):718-31.

12. Borer A, Gilad J, Meydan N, et al. Impact of active monitoring of infection control practices on deep sternal infection after openheart surgery. Ann Thorac Surg. 2001;72(2):515-20.

13. Hogan C, Lunney J, Gabel J, Lynn J. Medicare beneficiaries' costs of care in the last year of life. Health Aff (Millwood). 2001;20(4):188-95.

14. Gibson R, Janardan PS. The treatment trap. Chicago: Rowman \& Littlefield; 2010. 